\title{
Ischemic Stroke as Presenting Feature of Polycythemia Rubra Vera: A Rare Case Report
}

\author{
Dr Pradip Kumar Behera ${ }^{1}$, Dr K. P. Tripathy ${ }^{2}$, Dr Ranjita Panigrahi ${ }^{3}$ \\ ${ }^{1}$ Asso. Prof, Dept. of Medicine, Kalinga Institute of Medical Sciences (KIMS), Bhubaneswar, Odisha \\ ${ }^{2}$ Asso. Prof, Dept. of Medicine, Kalinga Institute of Medical Sciences (KIMS), Bhubaneswar, Odisha \\ ${ }^{3}$ Asso. Prof, Dept. of Pathology, Kalinga Institute of Medical Sciences (KIMS), Bhubaneswar, Odisha
}

\begin{abstract}
Polycythemia rubra vera (PRV) commonly presents with non specific symptoms and in due course can develop Stroke. But ischemic stroke as the initial presentation of PRV is rare. A 36 yr male presented to us with right sided hemiparesis and imaging revealed one recent infarct over left thalamo-ganglionic area. Evaluation for underlying cause proved him as a case of polcythemia rubra vera. The case is reported here in view of rarity.
\end{abstract}

Key Words:Hypercoagulable state, Ischaemic stroke, Polycythemia rubra vera,

\section{Introduction}

Stroke or cerebrovascular accident is defined as the abrupt onset of a neurological deficit that is attributable to a focal vascular cause ${ }^{1}$. It is the third leading cause of death worldwide and major cause of adult neurological disability. About $80 \%$ of strokes are caused by cerebral ischemia and rest are due to cerebral haemorrhage. ${ }^{2}$ Ischemia occurs when there is decrease in blood flow to less than $20 \mathrm{ml} / 100 \mathrm{~g}$ of brain tissue per minute. Reduction of blood flow to less than $16 \mathrm{ml} / 100 \mathrm{~g}$ of brain tissue per minute leads to tissue death within one hour. In the absence of blood flow, death of brain tissue can occur within 4 to 10 minutes ${ }^{3}$. Majority of ischemic strokes are athero-thrombtic and rest are embolic out of which around 20\% are cardioembolic. Also there are some rare causes of ischemic stroke which includes several haematological disorders and haemostatic defects. A common feature of these disorders is the development of a hypercoagulable state. Hematological diseases such as Essential Thrombocythemia, Polycythemia rubra vera and Thrmbotic thrombocytopenic purpura can cause stroke though rare ${ }^{4}$. Usually these disease conditions present with other symptoms and signs before development of stroke. Here we report a case of Polycythemia vera who presented to us with stroke without having any other symptoms before.

\section{Case Report:}

A 36 yrs nondiabetic, normotensive, Hindu male, farmer by occupation presented to us with sudden onset weakness of right upper and lower limbs, deviation of face to left and difficulty in speech of 3 days duration. There was no history of head trauma, seizure, loss of consciousness or similar episodes in past. He was apparently healthy prior to this illness without any history of chest pain, chest tightness, respiratory distress, skin rash, bleeding manifestations, itching, significant headache, blurring of vision, leg swelling or fever. $\mathrm{He}$ was married with two children, was non smoker and non alcoholic.

On clinical examination, he was conscious, well oriented, of average body built and nutritional status, Hight- $185 \mathrm{~cm}$, weight- $66 \mathrm{~kg}$, Pulse was $68 / \mathrm{min}$, regular without any specific character, Blood pressure was 130/80 $\mathrm{mm}$ of $\mathrm{Hg}$ in right arm, in supine position and there was prominent injected conjunctiva (Fig.1). There was no pedal edema, cyanosis, clubbing or lymphadenopathy. Skin, hair and nails were healthy ad no other significant abnormality detected on general examination.

Neurological examination revealed dysarthria, right sided upper motor type facial palsy, power on right upper and lower limbs was $3 / 5$ with brisk deep tendon jerks and plantar reflex was extensor on the same side. On the left upper and lower limbs power was $5 / 5$ with normal deep tendon reflexes and plantar response was flexor. On examination of abdomen, mild splenomegaly was detected. Liver was not palpable. There was no mass or evidence of free fluid in abdomen. Chest and cardiovascular system examination revealed no abnormality.

With the provisional diagnosis of cerebrovascular accident with right sided hemiparesis, thorough investigation was planned. NCCT scan of brain showed a hypodense lesion over left thalamo-ganglionic area suggestive of recent infarct(Fig 2). Further investigations were done to evaluate the underlying cause. Complete blood count(CBC) study revealed $\mathrm{Hb} \% 19.2$ gm\%(Ref. Range-13.5-17.5), total red blood cell count $5.72 \times 10^{12}$ /L(Ref. Range 4.5-5.5), Total white blood cell count $7.6 \times 10^{9} / \mathrm{L}$ with neutrophil $71 \%$, Total platelet count $495 \times 10^{9} / \mathrm{L}$ and packed cell volume(PCV) $52.01 \%$ (Ref range 40-50). Peripheral blood film showed neutrophilic leukocytosis without any premature cells.ESR was $05 \mathrm{~mm}$ in $1^{\text {st }}$ hour. Serum uric acid was $8.5 \mathrm{mg} / \mathrm{dl}$. On the 
other hand fasting plasma sugar, $\mathrm{HbA}_{1} \mathrm{C}$, fasting lipid profile, blood urea, serum creatinine and serum electrolytes were within normal range.ICT for malaria, HBsAg, Anti HCV were negative. Ultrasonography of whole abdomen showed splenomegaly without any focal lesion and all other organs were within normal limits. X-Ray of chest, ECG and 2D Echo. Of heart were normal.

With elevated RBC count, PCV, $\mathrm{Hb} \%$ and TPC, normal serum erythropoietin level and absence of any apparent secondary cause of erythrocytosis, the case was diagnosed as polycythmia vera. In a non diabetic, normotensive middle aged male(<40 yrs)without dyslipidemia, normal BMI and no other significant risk factors for atherothrombosis and no evidence of source of embolism polycythemia vera was thought to be the underlying cause of ischemic stroke. The case was as managed with antiplatelet agents. In consultation of the haematologist, venesection was done, Hydroxyuria 500mg/day and Allopurinl $300 \mathrm{mg} /$ day was started. He was discharged after one week and had satisfactory neurological recovery on follow up after one month.

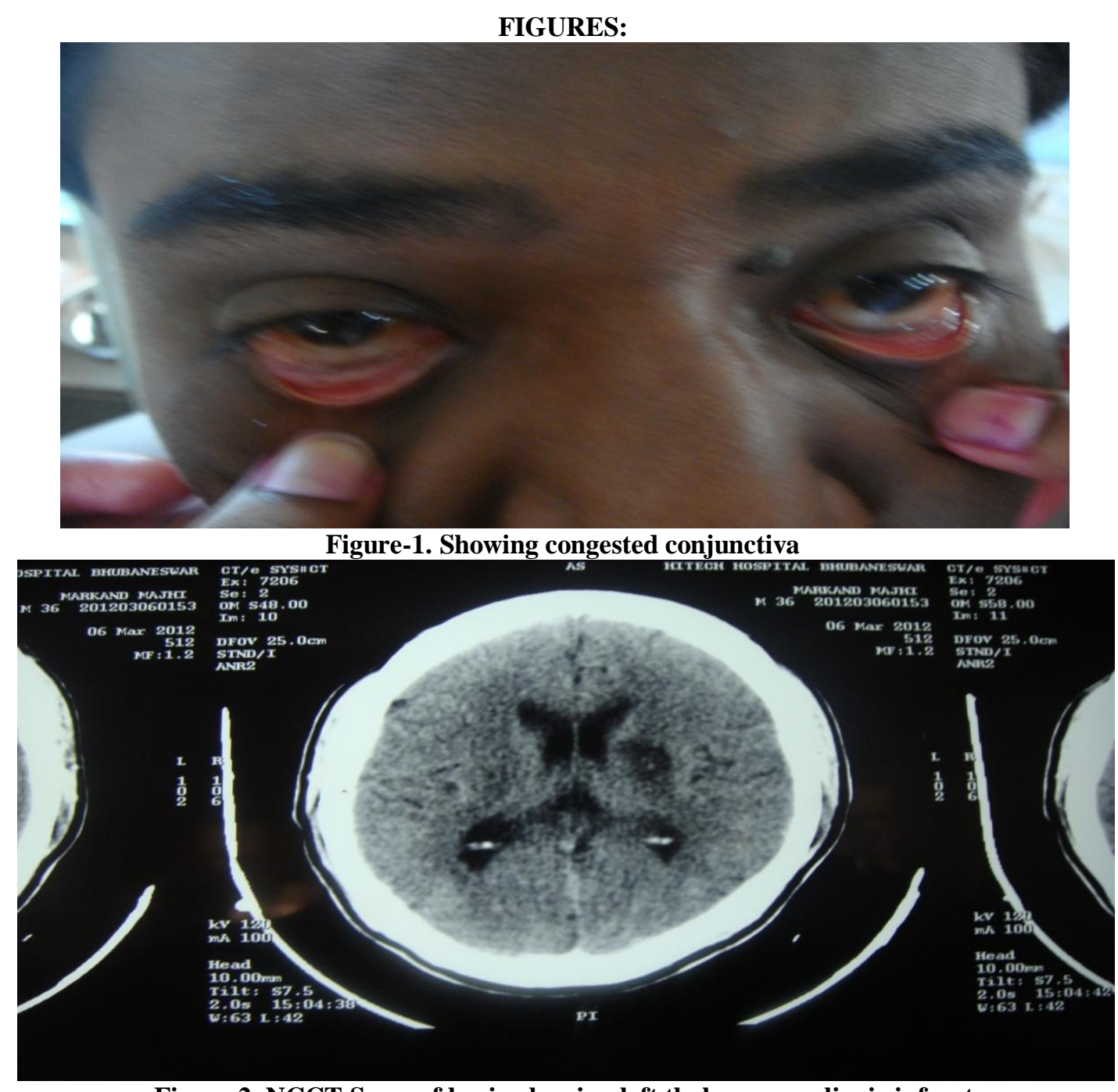

Figure 2. NCCT Scan of brain showing left thalamo-ganglionic infarct

\section{Discussion:}

Polycythemia vera is a clonal disorder involving a multipotent hematopoietic progenitor cell in which phenotypically normal red cells, granulocytes and platelets accumulate in absence of a recognisable physiologic stimulus. It is the most common type of myeloproliferative disorder.The patients of PCV may be asymptomatic or commonly present with nonspecific symptoms like fatigue, headache, dizziness, vertigo, tinnitus, pruritus (typically after a warm bath), dyspepsia or blurring of vision. Thrombotic phenomena are a frequent presentation including cerebral arterial and venous thrombosis causing stroke. The most likely mechanisms are hyperviscosity and impaired cerebral blood flow. An inverse relationship between cerebral blood flow and the packed cell volume has been shown in PRV. Cerebral blood flow significantly reduces in polycythemia, impairs normal uptake of oxygen by tissues and impairs microcirculation ${ }^{6}$. Moreover, an increased hematocrit has been associated with decreased reperfusion and increased infarct size following an 
ischemic stroke. Other neurological manifestations in PRV are transient ischemic attacks and cerebral haemorrhages. Less specific symptoms like parasthesia, vertigo, tinnitus , headache and visual disturbance are also explained on the basis of reduced cerebral blood flow. Fluctuating dementia, confusional states and chorea attributed to multiple small vessel occlusions in cortex and basal ganglia have also been recorded ${ }^{6}$.

Treatment of ischemic stroke with PRV should aim at reducing further clot formation by reducing high blood viscosity. Stroke secondary to PRV should be treated with standard stroke regimen as well as PRV therapy ${ }^{7}$.Serial phlebotomies are the mainstay of treatment, with aim to reduce hematocrit to $<45 \%$ in men, $<42 \%$ in women and $<36 \%$ in pregnancy ${ }^{8}$. Hydroxyuria is used as an adjunct to phlebotomy especially in patients prone to thrombosis ${ }^{9}$. Allopurinol can be used for high uric acid levels. Interferon is reserved of high risk women of child bearing potential and for refactorypruritus. Alkylating agents and radioactive phosphorous $32 \mathrm{P}$ should be considered only in elderly as they are highly leukemogenic. Low dose aspirin $(75-100 \mathrm{mg} / \mathrm{day})$ should be given to all petients if not otherwise contraindicated ${ }^{10}$. Hematocrit assay should be done as a routine in all stroke patients. Effective management of polycythemia rubra vera can prevent the recurrence of further stroke.

\section{Refferences:}

[1] Smith Wade S, English Joey D, Johnston S Claiborne. Cerebrovascular Diseases. Harrison's principles of internal medicine.18 ${ }^{\text {th }}$ Edn. (Mc Graw Hill Companies. 2012) 3270-3299.

[2] Hugh SM. Stroke-causes and clinical features. Medicine international 2005; 32:9, 05(1):36-40.

[3] Frizzell JP. Acute stroke - pathophysiology, diagnosis and treatment. AACN clinical issues 2005; 16(4):421-40.

[4] Matijevic N, Wu KK. Hypercoagulable states and strokes. Curr Atheroscler Rep 2006; 8(4): 324-29.

[5] Spivak Jerry L . Polycythemia vera and other myeloproliferative diseases. Harrison s principles of internal medicine.18 ${ }^{\text {th }}$ Edn. (Mc Graw Hill Companies. 2012) 898-904.

[6] Pearce JMS, Chandrasekera CP, Ladusans EJ. Lacunar infarcts in polycythemia with raised packed cell volume . BMJ 1983; 287:935-36.

[7] Meng R, Zhou J,J i XM et al. The diagnosis and treatment of polycythemia rubra vera manifesting as acute cerebral stroke. Zhonghua Nei Ke Za Zhi 2006; 45(5): 366-8.

[8] Spivak JL. Polycythemiavera. myths, mechanisms and management. Blood.2002; 100:4272-90.

[9] Fruchman SM, Mack K, Kaplan ME et al. From efficacy to safety. polycythemia vera study Group Report on Hydroxyuria in patients with polycythemia vera. Semin Hematology. 1997;34:17-23.

[10] Finazzi G. Risk stratification, staging and treatment of patients with Polycythemia Vera: Italian and Europian Collaberation on low dose aspirin in Polycythemia Vera.Semin Thromb Hemost. 2006;32:276-82. 